\title{
Protective effects of thalidomide on pulmonary injuries in a rat model of paraquat intoxication
}

\author{
Dan Li ${ }^{1,2}$, Xiao-Wei Zhang ${ }^{3}$, Xing-Quan Jiang ${ }^{1}$, Yong-Jie Yin ${ }^{1}$, Zhe Fan ${ }^{4}$, Cheng-Bo Sun ${ }^{5}$, Xing-Hai Chen ${ }^{1}$, \\ Yan-Hui Li ${ }^{1}$ and Ke-Xiang Liü ${ }^{2 *}$
}

\begin{abstract}
Background: This study was designed to evaluate the protective effects of thalidomide on paraquat (PQ)-induced lung injuries in a rat model and to explore the underlying mechanisms.

Methods: Rats were exposed to $50 \mathrm{mg} / \mathrm{kg}$ PQ by oral gavage, and treated with thalidomide through oral administration at $60 \mathrm{mg} / \mathrm{kg}$ once a day, 6 days/week for 2 weeks. Serum levels of IL-6, TNF-alpha, TGFbeta1 and COL1A1 were detected at different time points after paraquat exposure. At the end of the study, lung tissues were collected for pathological inspection as well as analyses of water content and expression levels of IL-6, TNF-alpha, TGFbeta1 and COL1A1 mRNA.

Results: The results showed that thalidomide treatment could significantly alleviate PQ-induced pathological changes in lung tissue and severity of lung edema. Thalidomide treatment after PQ exposure resulted in significantly reduced serum levels of IL-6, TNF-alpha, TGF-beta1 and COL1A1, as compared to PQ group. PCR analysis demonstrated that expression levels of IL-6, TNF-alpha, TGF-beta1 and COL1A1 in lung tissue were significantly increased after PQ exposure but reduced by thalidomide, which were confirmed by immunohistochemistry staining.
\end{abstract}

Conclusions: Our results indicated that inflammatory factors played important roles in PQ-induced lung injuries and thalidomide could protect rats from PQ-induced lung injuries by inhibiting the upregulation of inflammatory factors.

Keywords: Paraquat, Lung injury, Thalidomide, Protective effects

\section{Background}

Paraquat (1,1'-dimethyl-4,4'-bipyridilium dichloride, PQ) is a widely used nonselective herbicide around the world and the incidence rate of PQ intoxication has been reported to be increasing. The primary damages caused by $\mathrm{PQ}$ occur in lung tissue due to the accumulation of PQ. PQ exposure results in acute damage and destruction of alveolar epithelial cells, pulmonary edema, and infiltration of inflammatory cells within a few days. The acute injury phase is then followed by a final pulmonary fibrotic phase that lasts for several weeks featured by infiltration of myofibroblasts into the alveolar spaces and septa, and differentiation in fibroblasts with the production of collagen [1].

\footnotetext{
*Correspondence: 1183969122@qq.com

${ }^{2}$ Department of Cardiovascular Surgery, The Second Hospital of Jilin University, No. 218 Ziqiang Street, Changchun 130041, Nanguan District, People's Republic of China

Full list of author information is available at the end of the article
}

Pulmonary fibrosis is a major hallmark and a leading cause of death in PQ intoxication. The high mortality rate of PQ-intoxicated patients imposes a challenge to clinical practitioners due to the lack of an antidote or effective treatment to prevent pulmonary fibrosis [2].

A number of studies have suggested that the PQinduced oxidative stress is the primary mechanism for initiating lung damage by PQ. PQ intoxication generates numerous oxygen free radical species by cyclic oxidoreduction of $\mathrm{PQ}$, which cause disruptions of alveolar epithelial cells and Clara cells, infiltration of inflammatory cells into the interstitial and alveolar spaces, upregulation of several genes involved in inflammatory response, proliferation of fibroblasts, and deposition of excessive collagen $[1,3]$. Several inflammatory cytokines, particularly TNF- $\alpha$, IL- 6 , IL- $1 \beta$, and TGF- $\beta 1$ are found to play key roles in the pathogenesis of PQ-induced lung injury 
and fibrosis $[4,5]$. Although there is no effective therapy for PQ poisoning, anti-inflammatory drugs including corticosteroids and immunosuppressive drugs have been used in the clinical treatment for PQ intoxication $[6,7]$.

Thalidomide (Thal), originally developed in the 1950s as a tranquilizer, was discontinued for its potent teratogenic effects in the 1960s. It has recently being shown to have various pharmacological properties, including immunomodulation [8], anti-inflammation [9-11], and anti-angiogenesis [12]. Thalidomide and its analogs have been used in the treatment of a variety of disorders including erythema nodosum leprosum, multiple myeloma, rheumatoid arthritis, Crohn's disease, prostate cancer and lupus erythematosus [13]. In recent experimental studies, thalidomide has been demonstrated to have anti-fibrotic effects by suppressing the expression of IL-6, TGF- $\beta$, and angiogenesis-related growth factors that play a crucial role in the proliferation and differentiation of lung fibroblasts [14].

Based on the fact that inflammatory mediators contribute greatly to PQ-induced acute lung injury and subsequent fibrosis, we hypothesize that thalidomide may be potentially used to relieve PQ-induced pulmonary inflammation and fibrosis. In the present study, we explored whether Thal has protective effects on PQinduced lung injury and fibrosis using PQ intoxication rat model. Moreover, we also investigated the mechanisms underlying the therapeutic effect of thalidomide on pulmonary inflammation and fibrosis.

\section{Methods \\ Reagents}

Paraquat was provided by Shandong Yinuo Company (Shandong, China); Thalidomide was purchased from Changzhou Pharmaceutical Company (Changzhou, China); Polyclonal antibodies against TNF- $\alpha$, IL-6, TGF $\beta 1$ and collagen-1 were purchased from Boster Biological Technology (Wuhan, China); Horseradish peroxidase (HRP)-conjugated goat anti-rabbit secondary antibody and streptavidin-peroxidase immunohistochemistry kit were provided by Beijing Zhongshan-Golden Bridge Biological Technology (Beijing, China); Trizol and SuperScript II Reverse Transcriptase were obtained from Invitrogen (Carlsbad, USA); Taq DNA polymerase and DNA size marker were purchased from Beijing Dingguo Changsheng Biotechnology (Beijing, China).

\section{Animal model and experimental procedures}

All animal studies were performed in accordance with the guidelines of the Jilin University and the animals received human care in compliance with the Principles of Laboratory Animal Care. Healthy female Wistar rats weighing $250 \sim 300$ g purchased from Animal Research Center of Jilin University were hosted in a pathogen-free animal facility under a standard 12-h light/12-h dark cycle. Rats were fed standard rodent chow and water ad libitum.

Rats were randomly assigned to three groups: (1) normal control group, five rats with oral administration of $1 \mathrm{ml}$ of $0.9 \% \mathrm{NaCl}$ solution only; (2) PQ group, 14 rats with one time oral administration of paraquat $(50 \mathrm{mg} / \mathrm{kg})$ in $1 \mathrm{ml}$ of saline; and (3) PQ+ Thal group, ten rats with one time oral administration of paraquat $(50 \mathrm{mg} / \mathrm{kg})$ in $1 \mathrm{ml}$ of saline followed by oral gavage of thalidomide at $60 \mathrm{mg} / \mathrm{kg}$ once a day, 6 days/week for 2 weeks. Blood samples were collected through retro-orbital bleeding at days $1,2,3,5,7,10$ and 15 after paraquat exposure for detection of IL-6, TNF- $\alpha$, TGF $\beta 1$ and COL1A1 levels. Rats were euthanized at day 15 and lung tissues were collected for analyses of water content and expression levels of IL-6, TNF- $\alpha$ and COL1A1 mRNA in lung as well as pathological inspection.

Detection of serum IL- 6 , TNF- $\alpha$, TGF $\beta 1$ and COL1A1 levels The concentrations of IL-6, TNF- $\alpha$, TGF $\beta 1$ and COL1A1 were determined using ELISA. Briefly, 96-well, flat bottom plates were coated with serum samples overnight at $4{ }^{\circ} \mathrm{C}$. The plates were washed and blocked with PBS with $10 \%$ fetal bovine serum for $1.5 \mathrm{~h}$. After the plates were washed, primary detection antibodies specific to IL-6, TNF- $\alpha$, TGF $\beta 1$ and COL1A1 were added to the respective wells for $1.5 \mathrm{~h}$. The plates were washed and horseradish peroxidase (HRP)-conjugated secondary antibody was added to the plates for $1.5 \mathrm{~h}$. Following a series of stringent washing, the substrate reagent was added into each well and absorbance was read at $490 \mathrm{~nm}$ using the plate reader. The detection limits of ELISA methods for IL-6, TNF- $\alpha$, TGF $\beta 1$ and COL1A1 were all below $50 \mathrm{pg} / \mathrm{ml}$.

\section{Histological examinations of the lungs}

The lung tissues from each treatment group were excised, fixed in $10 \%$ buffered formalin and then embedded in paraffin. Sliced lung tissue sections $(2 \mu \mathrm{m}$ in thickness) were stained with Hematoxylin-and-Eosin $(H \& E)$ and pathological changes were examined by experienced pathologists. Masson's trichrome staining was used to assess collagen deposition in lung extracellular matrix.

\section{Detection of mRNA expression levels of IL- 6 , TNF- $a$, TGF $\beta 1$ and COL1A 1 in the lungs by Reverse Transcription-Polymerase Chain Reaction (RT-PCR)}

Total RNA from rat lung tissue was extracted using Trizol (Invitrogen, California, USA) by following manufacturer's instruction. cDNA was synthesized from $2 \mu \mathrm{g}$ of total RNA by using SuperScript II reverse transcription kit (Invitrogen, California, USA) for each sample. Conventional PCR was used to detect expression levels of IL-6, TNF- $\alpha$, TGF $\beta 1$ and COL1A1 with GAPDH used as endogenous control. The specific primer sets (Table 1) 
Table 1 Primers used for the PCR detection of specific gene expression

\begin{tabular}{|c|c|c|c|}
\hline Gene & Forward & Reverse & PCR product size \\
\hline TGF- $\beta 1$ & 5'-GACTCCTGCTGCTTT CTCC-3' & 5'-GCGGTCCACCATTA GCAC-3' & $531 \mathrm{bp}$ \\
\hline IL-6 & 5'-CCACGGCCTTCCCTA CTTC-3' & 5'-TTGGTCCTTAGCCAC TCCT-3' & $499 \mathrm{bp}$ \\
\hline COLIA1 & 5'-GTCCTATGGCTATGATG AGAA ATC-3' & 5'-CACCATCCAAACCA CTGAAAC-3' & $335 \mathrm{bp}$ \\
\hline TNF- $a$ & 5'-GTCCCCAAAGGGATGA GAAG T-3' & 5'-TGAGATAGCCAAATCG GCT GAC-3' & $323 \mathrm{bp}$ \\
\hline GAPDH & 5'-ACCACAGTCCATGCCAT CAC-3' & 5'-TCCACCACCCTGTTGCT GTA-3' & $452 \mathrm{bp}$ \\
\hline
\end{tabular}

were designed based on sequences deposited in the NCBI GenBank database. The thermal cycling conditions included 1 cycle at $94^{\circ} \mathrm{C}$ for 2 min and 35 cycles at $94{ }^{\circ} \mathrm{C}$ for $1 \mathrm{~min}, 50{ }^{\circ} \mathrm{C}$ for $1 \mathrm{~min}$ and $72{ }^{\circ} \mathrm{C}$ for $1 \mathrm{~min}$ followed by 1 cycle at $72{ }^{\circ} \mathrm{C}$ for $10 \mathrm{~min}$. PCR products were resolved by electrophoresis on $1.5 \%$ agarose gel and images were collected using gel imaging system.

\section{Immunohistochemistry detection of IL-6, TNF- $\alpha$, TGF $\beta 1$ and COL1A1 expression in the lungs}

Immunohistochemical staining (IHC) was performed using streptavidin-peroxidase immunohistochemistry kit by following the instructions. Rabbit polyclonal antibodies against TNF- $\alpha$, IL-6, TGF $\beta 1$ and COL1A1 were used as primary antibodies, and equal amount of purified rabbit IgG was used as negative control. The signal was developed using DAB substrate.

\section{Statistical analysis}

All data were presented as the mean \pm standard differences (S.D.). Data were analyzed by one-way analysis of variance (ANOVA) with Bonferroni post-tests for comparison between groups. In all cases, the difference was considered statistically significant as $P<0.05$.

\section{Results \\ Effects of thalidomide on PQ-induced morbidity and mortality}

The rats in PQ group presented with tachypnea, labored breathing, and anorexia within 2 to 3 days post PQ exposure. Two out of 14 rats died on day 2 and one died on day 3 after PQ administration. Severe interstitial edema, alveolar hemorrhage, and inflammation in all alveolar interstitial tissues and lumens were observed at necropsy (data not shown). In contrast, no apparent signs of respiratory distress were observed in rats in PQ + Thal group after PQ exposure and no animal died in this group.

\section{Effect of thalidomide on lung water content}

Lung water content was calculated by the formula of (lung wet weight-lung dry weight)/lung dry weight. Lung water content was higher in PQ group at day 15 post PQ exposure compared to control group and was significantly reduced in the PQ + Thal group compared to PQ group $(p<0.05)$ (Table 2$)$. This indicated that lung edema remained for a long period of time following PQ intoxication, and treatment with thalidomide could effectively relieve PQ-induced lung edema.

\section{Thalidomide alleviated the histopathological changes of lung caused by $P Q$}

PQ-induced lung structural changes and alleviative effects of thalidomide on PQ-induced damages are depicted in Fig. 1. Histological changes were assessed with H\&E staining and lung fibrosis was identified by using Masson's Trichrome stain for collagen. Animals from control group presented a normal pulmonary structure at light microscopy, without evidences of alveolar collapse, cellular infiltrations, or collagen accumulation. Paraquat intoxicated rat lung on day 15 showed diffuse alveolar damages with thickening of alveolar walls and widespread inflammation in alveolar spaces and septa. There were significant peribronchial and perialveolar deposition of collagen observed in Masson's trichrome staining. In comparison with the PQ group, the occurrence of the above, referred alterations were drastically attenuated in the PQ + Thal groups, particularly inflammation, hemorrhage, and the amount of accumulation of collagenous fiber.

\section{Effects of thalidomide on serum levels of IL-6, TNF-a, TGF- $\beta 1$ and COL1A1 induced by PQ}

Serum levels of IL-6, TNF- $\alpha$, TGF- $\beta 1$ and COL1A1 were measured by ELISA on day 1, 3, 5, 7, 10 and 15 after PQ exposure. In PQ group, serum IL-6 levels significantly elevated on day 1 compared to control group, peaked on day 3 , and gradually reduced thereafter. Thalidomide treatment resulted in accelerated reduction of PQinduced elevation of IL-6 levels (Fig. 2a).

\begin{tabular}{ll} 
Table 2 Water content of lung tissues at the end of experiment \\
\hline Group & Lung water content \\
\hline Normal control & $4.94 \pm 0.24$ \\
$P Q$ & $5.21 \pm 0.72$ \\
$P Q+$ Thal & $4.63 \pm 0.54^{*}$ \\
\hline
\end{tabular}

Data are presented as (Mean \pm SD)

${ }^{*} P<0.05$, compared to $\mathrm{PQ}$ 


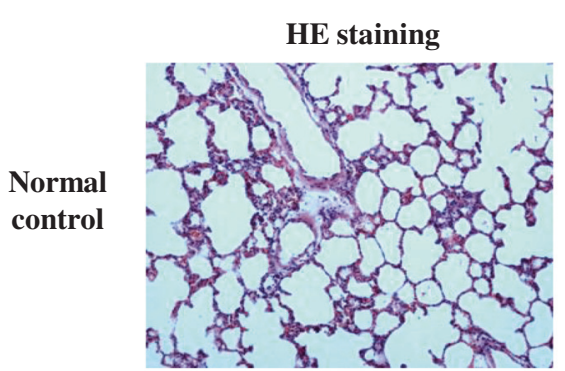

(a)

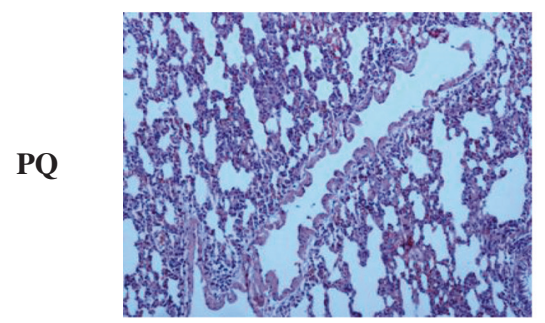

(c)

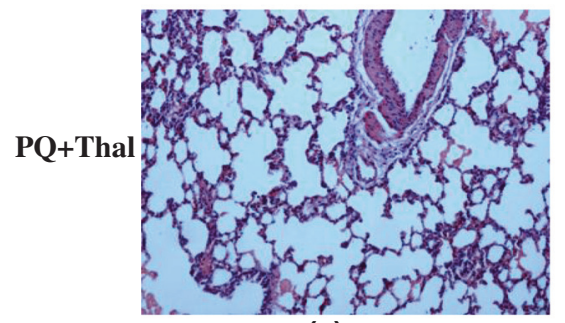

(e)

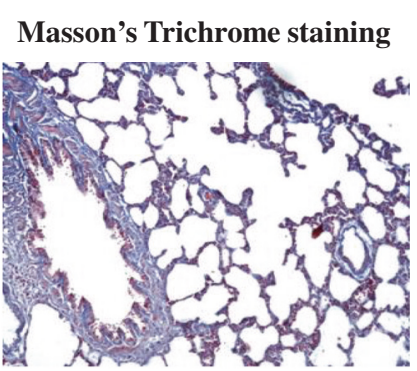

(b)

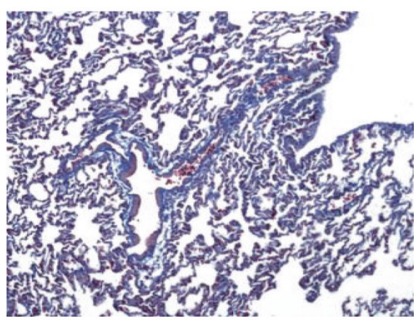

(d)

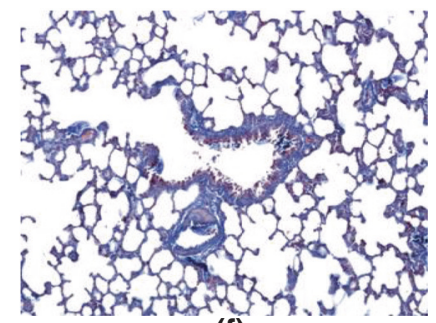

(f)

Fig. 1 Thalidomide alleviated PQ induced histopathological changes in lung tissues in rats. Lung tissues from Control (a, b), PQ (c, d) and PQ + Thal $(\mathbf{e}, \mathbf{f})$ groups were subjected to pathological examination on day 15 after PQ administration by hematoxylin and eosin staining $(\times 100)(\mathbf{a}, \mathbf{c}, \mathbf{e})$ and Masson's trichrome staining $(\times 100)(\mathbf{b}, \mathbf{d}, \mathbf{f})$

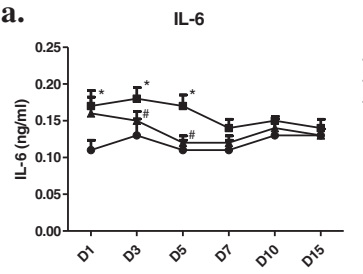

c.

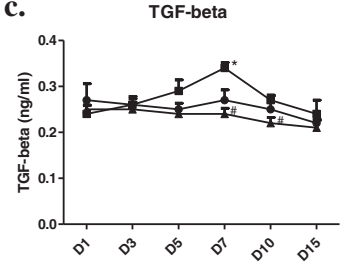

b.

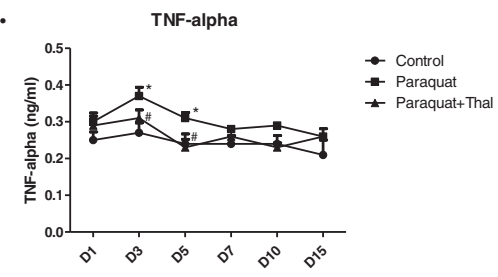

d.

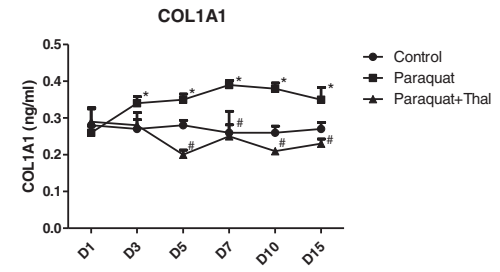

Fig. 2 Effects of thalidomide on serum levels of IL-6, TNF-a, TGF- $\beta 1$ and COL1A1 induced by PQ. Blood samples from rats from each treatment group were collected through retro-orbital bleeding at days 1, 2, 3, 5, 7, 10 and 15 after paraquat exposure and serum levels of IL-6 (a), TNF-a (b), TGF- $\beta 1$ (c) and COL1A1 (d) were measured by ELISA. The values represent mean \pm SD from each group $(\bullet$ Control, $-\mathrm{PQ}$ group and $\boldsymbol{\Delta} \mathrm{PQ}+\mathrm{Thal}$ group). * $p<0.05$ compared to Control, \# $p<0.05$ compared to Paraquat 
Serum TNF- $\alpha$ levels increased in PQ group on day 1 post PQ exposure, peaked on day 3, and remained higher than control group on day 5. Rats treated with thalidomide following PQ exposure had significantly reduced serum TNF- $\alpha$ levels on day 3 and 5 compared to PQ group (Fig. 2b).

PQ caused serum levels of TGF- $\beta 1$ to increase on day 5 and peak on day 7 after oral ingestion. However, no significant changes of serum TGF- $\beta 1$ levels were observed in rats treated with thalidomide after PQ exposure (Fig. 2c).

Serum COL1A1 levels in rats of PQ group significantly elevated on day 3 , peaked on day 7 and remained higher than control on day 15 . Thalidomide treatment completely reversed the PQ-induced increase of COL1A1 levels (Fig. 2d).

Effects of thalidomide on mRNA expression levels of IL-6, TNF- $\alpha$, TGF- $\beta 1$ and COL1A1 in lung tissues after PQ intoxication

Expression levels of IL-6, TNF- $\alpha$, TGF- $\beta 1$ and COL1A1 mRNA transcripts in lung tissues were examined using RT-PCR on day 15 after PQ administration (Fig. 3). Expressions of IL- 6 and TNF- $\alpha$ mRNA were significantly up-regulated in lung tissues in PQ group compared to control, indicating the involvement of inflammatory cytokines in PQ-induced pathological changes of lung. In addition, expressions of TGF- $\beta 1$ and COL1A1, two important fibrosis factors, were also significantly elevated at mRNA level in lung tissues at 15 days after PQ exposure. However, expressions of IL- 6, TNF- $\alpha$, TGF- $\beta 1$ and COL1A1 transcripts were all significantly lower in lung tissues from rats treated with thalidomide after PQ exposure compared to PQ group. These results indicate that thalidomide may prevent PQ-induced pulmonary fibrosis through downregulating the expression of TGF- $\beta$ 1 , TNF- $\alpha$ and IL-6 in lung tissues.

\section{Immunohistochemistry detection of the expression of IL-6, TNF- $\alpha$, TGF- $\beta 1$ and COL1A 1 proteins in lung tissues after $P Q$ intoxication}

Expression of IL-6, TNF- $\alpha$, TGF- $\beta 1$ and COL1A1 proteins in lung tissues were examined using immunohistochemical staining on day 15 after PQ administration. As shown in Fig. 4, there were weak and diffused signals of IL- 6 and TNF- $\alpha$ on vascular walls and alveolar walls in normal lung tissues from control group. Enhanced signals of IL-6 and TNF- $\alpha$ stainings were observed on alveolar walls in lung tissues from PQ group on day 15 post PQ exposure and thalidomide treatment significantly reduced IL- 6 and TNF- $\alpha$ expression in lung tissues. Expression of TGF- $\beta 1$, which was detected at low levels on alveolar walls in normal lung tissues, was increased in lung tissues from PQ group and significantly reduced by thalidomide treatment (Fig. 4). In normal lung tissues, COL1A1 is mainly detected on vascular walls and weakly expressed on alveolar walls. Although expression of COL1A1 in alveolar walls was dramatically

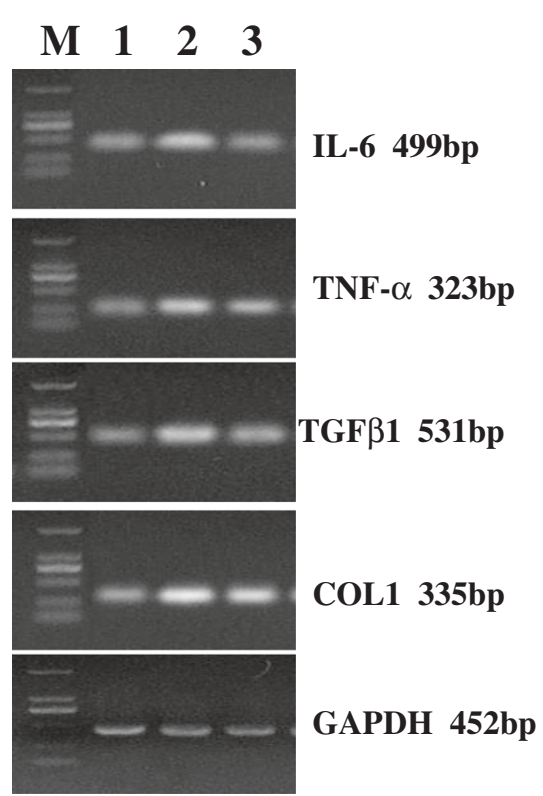

Lane M: DNA marker

Lane 1: Control

Lane 2: Paraquat

Lane 3: Paraquat+Thal

Fig. 3 Effects of thalidomide on expression levels of IL-6, TNF-a, TGF- $\beta 1$ and COL1A1 in lung tissues after PQ intoxication. Lung tissues from each treatment group were excised on day 15 after PQ administration and mRNA expression levels of IL-6, TNF-a, TGF- $\beta 1$ and COL1A1 genes were examined using RT-PCR 


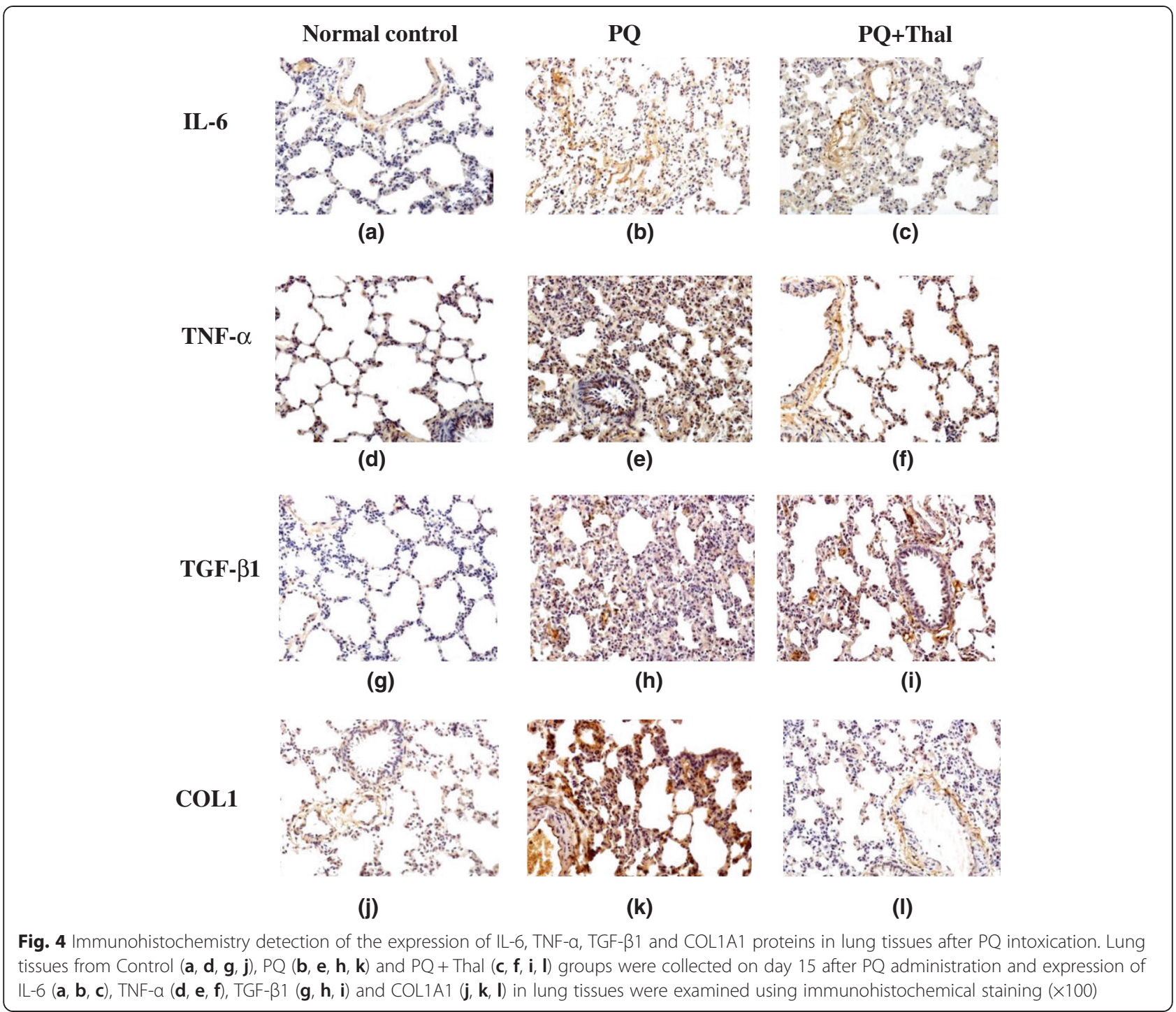

increased after PQ exposure, thalidomide treatment resulted in significantly decreased COL1A1 expression in lung tissues compared to PQ group.

\section{Discussion}

Intoxication of paraquat, a highly toxic quaternary ammonium herbicide, has a high mortality rate and poor prognosis due to severe acute lung injury and subsequent pulmonary fibrosis [15]. In this study we have demonstrated that thalidomide has a protective effect against PQ-induced lung injury and pulmonary fibrosis in rats. Our results showed that administration of thalidomide at $60 \mathrm{mg} / \mathrm{kg}$ could significantly reduce the degree of lung edema and pulmonary fibrosis caused by PQ in rats. Moreover, our data also suggested that the protective effect of thalidomide against PQ-induced pulmonary fibrosis was associated with decreased expression levels of inflammatory factors such as TGF- $\beta 1$, TNF- $\alpha$ and IL-6 in lung tissues after PQ exposure.

Lung is one of the major organs affected by PQ during PQ intoxication due to the accumulation of PQ in the lung through the active polyamine uptake system in the membrane of alveolar cell $[1,3]$. Although the molecular mechanism of $\mathrm{PQ}$-induced lung injuries is not fully understood, oxidative and inflammatory mediators have been found to play important roles in this process. It has become increasingly clear that reactive oxygen species generated through the cyclic oxido-reduction of PQ can not only result in tissue damages directly but also initiate and promote inflammatory responses through upregulation of inflammatory cytokines and adhesion molecules [16-18]. Such inflammatory cytokines, particularly IL-6, TNF- $\alpha$, TGF- $\beta 1$ are found to be involved in the pathogenesis of $\mathrm{PQ}$-induced pulmonary inflammation and 
subsequent fibrosis $[4,19,20]$. IL- 6 and TNF- $\alpha$ are cytokines that are released in both early acute inflammatory and late fibrotic phases of PQ-induced lung injury [4]. TGF- $\beta 1$ as the most important fibrogenic growth factor has been shown to stimulate fibroblast proliferation, matrix protein production and collagen synthesis [21]. Increased production of TGF- $\beta 1$ from lung injury caused by PQ has been associated with inflammation and fibrosis of lung tissue $[19,20]$. In this study, we observed that the serum levels of IL- 6 and TNF- $\alpha$ quickly elevated on day 1 , peaked on day 3 and remained higher than control on day 5 after PQ exposure in rats. However, serum TGF- $\beta 1$ level was not increased until day 5 and peaked on day 7 after PQ exposure. In addition, immunohistochemistry detection for the expression of IL-6, TNF- $\alpha$ and TGF- $\beta 1$ in lung tissues from rats on day 15 after PQ intoxication showed significant overexpression of these factors in lung tissues compared to control normal lung tissues. Our results, consistent with other studies $[4,19,20]$, demonstrate that inflammatory factors play important roles in PQ-induced lung injuries with proinflammatory factors IL- 6 and TNF- $\alpha$ involved in both acute and chronic phases and TGF- $\beta 1$ mainly in late fibrotic phase of PQ-induced lung injury.

Thalidomide and its analogs have been used in clinic as immunomodulatory drugs with anti-inflammatory, anti-proliferative and anti-angiogenic activities. It has been shown that thalidomide exhibits anti-inflammatory and anti-fibrotic activities by suppressing the production of proinflammatory cytokines and growth factors that play important roles in the tissue destruction and fibrosis in chronic inflammatory situations [19, 22, 23]. Several studies using animal models have demonstrated that thalidomide has therapeutic effects for fibrotic and inflammatory disorders such as bleomycin-induced lung fibrosis [14], peritonitis [23], pancreatitis [24], experimental diabetes [22] and liver cirrhosis [25]. Moreover, thalidomide was recently shown to protect against PQ-induced lung injury through different mechanisms in $\mathrm{PQ}$-intoxication mouse models [26-28]. Amirshahrokhi [26] showed that 6 days treatment of thalidomide in PQ intoxicated mice resulted in decreased production of inflammatory and fibrogenic cytokines including TNF- $\alpha$, IL-1 $\beta$, IL- 6 , and TGF- $\beta 1$ as well as reduced myeloperoxidase (MPO), nitric oxide (NO), and hydroxyproline content in lung tissues. In another two published studies performed by a Chinese group [27, 28], thalidomide was shown to attenuate PQinduced acute lung injury through multiple mechanisms which involved downregulation of TNF- $\alpha$, IL-1 $\beta$, and IL-6 via inhibition of NF- $\mathrm{kB}$ activation as well as protection from $\mathrm{PQ}$-induced lipid peroxide damage by activation of the Nrf2-ARE signaling pathway. In the current study we employed a 15-day thalidomide treatment regimen in the PQ intoxication rat model to evaluate the effects and potential mechanisms of thalidomide on PQ-induced lung injury, especially pulmonary fibrosis subsequent to acute lung injury phase. Our results showed that thalidomide treatment after PQ administration resulted in improved survival and rats treated with thalidomide had significantly reduced lung edema and improved pulmonary function at 15 days after treatment. Histological analysis showed that PQ-induced lung injuries characterized by a marked thickening of the alveolar septum and infiltration of various inflammatory cells were attenuated by administration of thalidomide. Lung fibrosis and collagen deposition caused by PQ was significantly relieved after 15-day thalidomide treatment as indicated by Masson's Trichrome staining. To explore the mechanisms of the protective effect of thalidomide on PQ-induced lung injury, we examined the expression levels of several inflammatory factors including TNF- $\alpha$, IL- 6 and TGF- $\beta 1$ that play important roles in pathogenesis of PQ-induced lung damages in both blood and lung tissues. Our results showed that blood levels of TNF- $\alpha$ and IL- 6 elevated to lower levels and reduced to normal in a shorter time after PQ exposure in rats treated with thalidomide as compared to control PQ intoxicated rats without thalidomide treatment. TGF- $\beta 1$ as one of the major fibrogenic factors in PQ-induced pulmonary fibrosis was significantly upregulated in blood at day 5 after PQ exposure, while thalidomide treatment completely reversed $\mathrm{PQ}$-induced increase of TGF- $\beta 1$ level in blood. Similarly, increase of blood COL1A1 level due to PQ exposure was also corrected by thalidomide treatment. These results indicated that immediate treatment with thalidomide after PQ intoxication could interfere with the onset of inflammatory and fibrogenic process and therefore significantly ameliorate pulmonary fibrosis. In addition, immunohistochemistry staining and PCR detection for expression of TNF- $\alpha$, IL- 6 and TGF- $\beta 1$ in lung tissues on day 15 after PQ intoxication further confirmed that thalidomide could significantly reduce the upregulation of the expression of these factors induced by $\mathrm{PQ}$ at both protein and RNA levels. Our findings together with others [26-28] indicate that thalidomide may exhibit anti-inflammatory and anti-fibrosis effects in a nonspecific way by regulating the expression of several key inflammatory factors, such as TNF- $\alpha$, IL- 6 and TGF- $\beta 1$. However, more sophisticated studies are needed to further identify potential signaling pathways and molecular mechanisms of thalidomide's anti-inflammatory and antifibrosis effects against PQ-induced lung injury.

\section{Conclusions}

Thalidomide exhibits protective effect against PQinduced acute lung injury and subsequent fibrosis by inhibiting the upregulation of inflammatory factors, particularly IL-6, TNF- $\alpha$ and TGF- $\beta 1$ in lung. Since PQ- 
induced lung damage begins at a very early stage of PQ poisoning, the treatment with thalidomide should be applied as early as possible after PQ exposure. Our results support clinic trials to explore whether adding thalidomide to current treatment regimen can improve the outcomes of patients with PQ intoxication.

\section{Abbreviations}

PQ: Paraquat; Thal: Thalidomide; TNF-a: Tumor necrosis factor-alpha; IL-6: Interleukine-6; IL-1 $\beta$ : Interleukine-1 beta; TGF- $\beta 1$ : Transforming growth factor-beta 1; H\&E: Hematoxylin-and-eosin; COL1A1: Collagen type I, alpha 1.

\section{Competing interests}

The authors declare that they have no competing interests.

\section{Authors' contributions}

$\mathrm{K}-\mathrm{XL}$ and $\mathrm{Y}-\mathrm{HL}$ conceived the study and designed the experiments. X-WZ, ZF and C-BS carried out the histologic study and RT-PCR. DL performed the ELISA, conceived of the study, and participated in its design and coordination and wrote the manuscript. X-QJ, Y-JY participated in data analysis and interpretation. $X-H C$ participated in the immunohistochemical study. All authors read and approved the final manuscript.

\section{Acknowledgements}

This work was supported by a research grant from the Science and Technology Agency of Jilin Province (20110720), Jilin, China.

\section{Author details}

'Department of Emergency Medicine, The Second Hospital of Jilin University, No. 218 Ziqiang Street, Changchun 130041, Nanguan District, People's Republic of China. ${ }^{2}$ Department of Cardiovascular Surgery, The Second Hospital of Jilin University, No. 218 Ziqiang Street, Changchun 130041, Nanguan District, People's Republic of China. ${ }^{3}$ Department of Clinical Laboratory, Children's Hospital of Changchun City, No. 69 Beian Road, Changchun 130061, Chaoyang District, People's Republic of China. ${ }^{4}$ Department of Technology, People's Procuratorate of Jilin Province, No. 1333 Jingyang Road, Changchun 130062, Lvyuan District, People's Republic of China. ${ }^{5}$ Department of Experimental Pharmacology and Toxicology, School of Pharmacy, Jilin University, No. 1163 Xinmin Avenue, Changchun 130021, Chaoyang District, People's Republic of China.

Received: 26 January 2015 Accepted: 23 July 2015

Published online: 28 July 2015

\section{References}

1. Tomita M, Okuyama T, Katsuyama H, Miura Y, Nishimura $Y$, Hidaka $K$, et al. Mouse model of paraquat-poisoned lungs and its gene expression profile. Toxicology. 2007;231(2-3):200-9. doi:10.1016/j.tox.2006.12.005.

2. Hwang KY, Lee EY, Hong SY. Paraquat intoxication in Korea. Arch Environ Health. 2002;57(2):162-6. doi:10.1080/00039890209602931

3. Ahmed AA. Protective effect of montelukast on paraquat-induced lung toxicity in rats. Biosci Trends. 2009;3(2):63-72.

4. Xiangdong J, Ming L, Yijing Z, Yanjun R, Guangran G, Hong S, et al. Role of growth factors in acute lung injury induced by paraquat in a rat model. Hum Exp Toxicol. 2011;30(6):460-9. doi:10.1177/0960327110372648.

5. Satomi Y, Tsuchiya W, Mihara K, Ota M, Kasahara Y, Akahori F. Gene expression analysis of the lung following paraquat administration in rats using DNA microarray. J Toxicol Sci. 2004;29(2):91-100.

6. Gawarammana IB, Buckley NA. Medical management of paraquat ingestion. Br J Clin Pharmacol. 2011;72(5):745-57. doi:10.1111/j.1365-2125.2011.04026.x.

7. Wu WP, Lai MN, Lin CH, Li YF, Lin CY, Wu MJ. Addition of immunosuppressive treatment to hemoperfusion is associated with improved survival after paraquat poisoning: a nationwide study. PLoS One. 2014;9(1):e87568. doi:10.1371/journal.pone.0087568.

8. Koch HP. Thalidomide and congeners as anti-inflammatory agents. Prog Med Chem. 1985;22:165-242.

9. Sampaio EP, Sarno EN, Galilly R, Cohn ZA, Kaplan G. Thalidomide selectively inhibits tumor necrosis factor alpha production by stimulated human monocytes. J Exp Med. 1991;173(3):699-703.
10. Haslett PA, Corral LG, Albert M, Kaplan G. Thalidomide costimulates primary human T lymphocytes, preferentially inducing proliferation, cytokine production, and cytotoxic responses in the CD8+ subset. J Exp Med. 1998;187(11):1885-92.

11. Corral LG, Haslett PA, Muller GW, Chen R, Wong LM, Ocampo CJ, et al. Differential cytokine modulation and T cell activation by two distinct classes of thalidomide analogues that are potent inhibitors of TNF-alpha. J Immunol. 1999;163(1):380-6.

12. D'Amato RJ, Loughnan MS, Flynn E, Folkman J. Thalidomide is an inhibitor of angiogenesis. Proc Natl Acad Sci U S A. 1994;91(9):4082-5.

13. Melchert M, List A. The thalidomide saga. Int J Biochem Cell Biol. 2007;39(7-8):1489-99. doi:10.1016/j.biocel.2007.01.022.

14. Tabata C, Tabata R, Kadokawa Y, Hisamori S, Takahashi M, Mishima M, et al. Thalidomide prevents bleomycin-induced pulmonary fibrosis in mice. J Immunol. 2007;179(1):708-14.

15. Yin $Y$, Guo $X$, Zhang SL, Sun CY. Analysis of paraquat intoxication epidemic (2002-2011) within China. Biomed Environ Sci. 2013;26(6):509-12. doi:10.3967/0895-3988.2013.06.014.

16. Tolando R, Jovanovic A, Brigelius-Flohe R, Ursini F, Maiorino M. Reactive oxygen species and proinflammatory cytokine signaling in endothelial cells: effect of selenium supplementation. Free Radic Biol Med. 2000;28(6):979-86.

17. Haddad JJ, Land SC. Redox signaling-mediated regulation of lipopolysaccharide-induced proinflammatory cytokine biosynthesis in alveolar epithelial cells. Antioxid Redox Signal. 2002;4(1):179-93. doi:10.1089/152308602753625942.

18. Lorne E, Zmijewski JW, Zhao X, Liu G, Tsuruta Y, Park YJ, et al. Role of extracellular superoxide in neutrophil activation: interactions between xanthine oxidase and TLR4 induce proinflammatory cytokine production. Am J Physiol Cell Physiol. 2008;294(4):C985-93. doi:10.1152/ajpcell.00454.2007.

19. Ishida Y, Takayasu T, Kimura A, Hayashi T, Kakimoto N, Miyashita T, et al. Gene expression of cytokines and growth factors in the lungs after paraquat administration in mice. Leg Med. 2006;8(2):102-9. doi:10.1016/j.legalmed.2005.08.010.

20. Kan B, Jian X, Zhou Q, Wang J, Yu G, Sun J, et al. Effect of transforming growth factor-beta1 on acute lung injury caused by paraquat. Mol Med Reports. 2014;9(4):1232-6. doi:10.3892/mmr.2014.1938.

21. Kovacs EJ, DiPietro LA. Fibrogenic cytokines and connective tissue production. FASEB J. 1994;8(11):854-61.

22. Amirshahrokhi K, Ghazi-Khansari M. Thalidomide attenuates multiple low-dose streptozotocin-induced diabetes in mice by inhibition of proinflammatory cytokines. Cytokine. 2012;60(2):522-7. doi:10.1016/j.cyto.2012.07.029.

23. Mondello S, Mazzon E, Di Paola R, Crisafulli C, Mondello P, Buemi M, et al. Thalidomide suppresses sclerosing encapsulating peritonitis in a rat experimental model. Shock. 2009;32(3):332-9. doi:10.1097/SHK.0b013e3181994974.

24. Malleo G, Mazzon E, Genovese T, Di Paola R, Muia C, Crisafulli C, et al. Effects of thalidomide in a mouse model of cerulein-induced acute pancreatitis. Shock. 2008;29(1):89-97.

25. Paul SC, Lv P, Xiao YJ, An P, Liu SQ, Luo HS. Thalidomide in rat liver cirrhosis: blockade of tumor necrosis factor-alpha via inhibition of degradation of an inhibitor of nuclear factor-kappaB. Pathobiology. 2006;73(2):82-92. doi:10.1159/000094492.

26. Amirshahrokhi K. Anti-inflammatory effect of thalidomide in paraquatinduced pulmonary injury in mice. Int Immunopharmacol. 2013;17(2):210-5. doi:10.1016/j.intimp.2013.06.005.

27. Li D, Xu LY, Chang ZJ, Zhao GJ, Nan C, Lu ZQ. EEffect of thalidomide in a mouse model of paraquat-induced acute lung injury and the underlying mechanisms]. Zhonghua Lao Dong Wei Sheng Zhi Ye Bing Za Zhi. 2013;31(3):178-83.

28. Zhao G, Cai X, She X, Li D, Hong G, Wu B, et al. [Effect of thalidomide in a mouse model of paraquat-induced acute lung injury and the underlying mechanisms]. Zhonghua Lao Dong Wei Sheng Zhi Ye Bing Za Zhi. 2014;32(11):806-12. 